\title{
Aprendizaje mediante el ejercicio práctico de actividades en asignaturas de ciencias agrarias
}

\author{
Borja Velázquez Martía, Isabel López Cortés ${ }^{b}$, Viviana Vanessa Vinueza Villarés ${ }^{\mathrm{a}}$, \\ Domingo Salazar-Hernández \\ ${ }^{a}$ Departamento de Ingeniería Rural y Agroalimentaria, Universitat Politècnica de València. Camino \\ de Vera s/n, 46022 Valencia (España), borvemar@dmta.upv.es, bepartamento de Producción \\ Vegetal, Universitat Politècnica de València. Camino de Vera s/n, 46022 Valencia (España), \\ islocor@upv.es
}

\section{Resumen}

En este trabajo se presenta la experiencia de la aplicación de una metodología de enseñanza-aprendizaje basada en prácticas en asignaturas de temática agrícola y agroindustrial. Ésta consistió en dedicar un $70 \%$ de las horas a prácticas de campo y laboratorio, y un 30\% de contenidos teóricos. Los contenidos teóricos fueron dirigidos a lecturas de bibliografía que después se exponen en foros antes de la aplicación de la práctica. Las prácticas se centraron en experimentar procesos propios de cada cultivo, tales como diferenciación de especies, técnicas de siembra y plantación, reconocimiento de plagas y enfermedades, técnicas de poda, realización de injertos etc. En las asignaturas de agroindustria, tales como aprovechamiento energético de la biomasa, las practicas se orientaron a ejercicios de laboratorio para calcular proiedades de materiales biocombustibles, tales como el poder calorifico, composición de CHN, cenizas etc. La evaluación se realiza de forma tradicional, mediante exámenes parciales y final. También se evalúan las memorias de prácticas. Los resultados obtenidos durante dos años mostraron un aumento de la puntuación de la valoración de la asignatura en las encuestas institucionales. El nivel de aprobados por curso aumentó alrededor de un $10 \%$. Además se realizaron entrevistas para indagar en los aspectos relevantes de la técnica, realizando los alumnos análisis DAFO (Debilidades, Amenazas, Fortalezas, Oportunidades). Entre las debilidades más relevantes se muestra la exigencia de ir a clase para seguir bien el método diseñado de aprendizaje.

Palabras clave: Prácticas, docencia inversa, evaluación continua . 


\section{Introducción}

El proceso de enseñanza-aprendizaje de asignaturas de temática agrícola requiere de forma general utilizar técnicas prácticas, donde los alumnos pueden experimentar procesos propios de cada cultivo, tales como diferenciación de especies, técnicas de siembra y plantación, reconocimiento de plagas y enfermedades, técnicas de poda, realización de injertos etc. Del mimo modo, en asignaturas técnicas del área agroindustrial, tales como el aprovechamiento energético de la biomasa, involucra conocimientos que en el ámbito profesional son también de índole práctico. A lo largo de los años se ha comprobado que son necesarios enfoques prácticos para la asimilación de los procesos productivos (Rodríguez-Cepeda, 2016). También se ha probado en la enseñanza de otras ramas del conocimiento tales como en ciencias de la salud (Pulido et al., 2008; Sánchez Rodríguez, 2017; Long et al., 2020). Esto obliga planificar los planes de estudios con un mayor número de horas de campo y laboratorio, simultaneadas con el contenido teórico en las aulas (Burke y Manathunga, 2020). Así queda reflejado en los procesos de renovación docente de las enseñanzas universitarias, necesaria para producir el cambio educativo. La docencia universitaria propicia el entorno para el desarrollo de tales dinámicas (Arandia, 2010; Dollinger, 2020). La idea de esta forma de enseñanza es dar mas peso al saber hacer cosas, que conocer y poseer conocimientos teóricos (Jiménez et al., 2007; Tsai et al., 2020). En relación al marco teórico esta metodología posibilita que los egresados en su ejercicio práctico desarrollen una comprensión explícita de su propias capacidades, adquiriendo confianza en sus conocimientos de base (Towers et al, 2020). Esto les proporciona una mayor seguridad, que les permite orientarse hacia capacidades de orden superior (funciones metacognitivas), tales como la práctica reflexiva y la conciencia de las propias capacidades para resolver problemas (Hundertmark y Schanze, 2020; Austin y King, 2020). De esta forma desarrolla una actitud en el que adquiere protagonismo el autoaprendizaje.

En principio la metodología evaluada permite el desarrollo de las capacidades de los egresados en lost res ámbitos: las que se refieren a saber como" (conocimiento procedimental), las que se refireren a eonocer acerca de" (conocimiento conceptual), las que se refieren a adquirir las cualidades personales o aptitudes y a las funciones metacognitivas que describen la combinación e integración de capacidades multiples.

Por ejemplo, en horticultura existe una diferencia entre concocer el proceso y tipos de siembra o injerto, y saber sembrar o injertar. Del mismo modo, existe diferencia en el saber lo que és el poder calorífico o el contenido de cenizas en un biocombustible, y saber realizar el análisis para su obtención. Además el proceso de aprendizaje de lo práctico fomenta el interés por asentar los conocimientos teóricos, es decir, anima a burcar respuestas en un proceso de autoaprendizaje (Mikelli, 2020). 
En este trabajo se presenta un estudio donde varias asignaturas de agronomía se enseñan dedicando un $70 \%$ de las horas a prácticas de campo y laboratorio, y un 30\% de contenidos teóricos. Los contenidos teóricos son dirigidos a lecturas de bibliografía que después se exponen en foros antes de la explicación de la práctica. Se pretende evaluar la percepción que tienen los propios estudiantes sobre el proceso de enseñanza así planteado, detectar inconvenientes para definir las pautas idoneas para la aplicación de esta técnica en el ámbito universitario.

\section{Materiales y métodos}

En este trabajo se presenta una valoración de la docencia en varias asignaturas de agronomía del Grado de Ingeniería Agronómica y del Medio Rural donde se ha aplicado un $70 \%$ de las horas a prácticas de campo y laboratorio, y un 30\% de contenidos teóricos. Los contenidos teóricos son dirigidos a lecturas de bibliografía que después se exponen en foros antes de la explicación de la práctica.

Cada práctica comienza con una discusión sobre la mejor técnica para realizar cada tarea de acuerdo a las características del cultivo. Generalmente se finalizaba la sesión con una presentación con la síntesis de los aspectos más importantes, realizada por el profesor. Finalmente se explica cómo ejecutar la tarea y posteriormente se debe realizar una memoria de la misma indicando los contenidos teóricos en los que se basa la solución adoptada.

La evaluación se realiza de forma tradicional, mediante exámenes parciales y final. También se evalúan las memorias de prácticas. Además se realizaron entrevistas para indagar en los aspectos relevantes de la técnica, realizando los alumnos análisis DAFO (Debilidades, Amenazas, Fortalezas, Oportunidades).

Para la realización del análisis DAFO se prepararon distintas preguntas abiertas organizadas en cuatro categorías sobre las que queríamos indagar. Su descripción se muestra en la Tabla 1 . 
Tabla 1.Categorias de evaluación a traves del análisis DAFO

\begin{tabular}{|l|l|}
\hline Categoría & Descripción \\
\hline Organización & $\begin{array}{l}\text { Se refiere al conocimiento de la estructuración de los contenidos en las unidades } \\
\text { didátricas, cronograma, materiales didácticos disponiles, y Sistema de evaluación } \\
\text { desde el comienzo de la asignatura }\end{array}$ \\
\hline Metodología & $\begin{array}{l}\text { Hace referencia al desarrollo de la prácticas. Desarrollo de los foros de discusión de } \\
\text { los conocimientos teóricos asociados a la aplicación de las prácticas. ¿La } \\
\text { metodología consigue la adquisición de conocimientos vinculados a la asignatua? }\end{array}$ \\
\hline Motivación & $\begin{array}{l}\text { Valoración de la vinculación de las prácticas a la motivación por la asignatura. ¿El } \\
\text { desarrollo de la metodología cumple con las espectativas tenidas por los alumnos } \\
\text { antes de cursar la asignatura? }\end{array}$ \\
\hline $\begin{array}{l}\text { Sistema de } \\
\text { evaluación }\end{array}$ & \begin{tabular}{l} 
Valoración del sistema de evaluación y su vinculación a los contenidos aprendidos. \\
\hline
\end{tabular}
\end{tabular}

\section{Resultados y discusión}

Los resultados obtenidos durante dos años mostraron un aumento de la puntuación de la valoración de la asignatura en las encuestas institucionales. En la Figura 1 se muestran las medias de las calificaciones obtenidas en las asignaturas en los años que se desarrolló la metodología práctica, 2017 y 2018 de color rojo, y la media de las valoraciones de las distintas asignaturas en los dos cursos anteriores, 2015 y 2016 de color azul, donde se aplicó una metodología convencional basadoa mayotitariamente en clases teóricas en aula. Se puede obserbar un incremento de la valoración del $20 \%$ en los años en los que se aplicó la metodología.

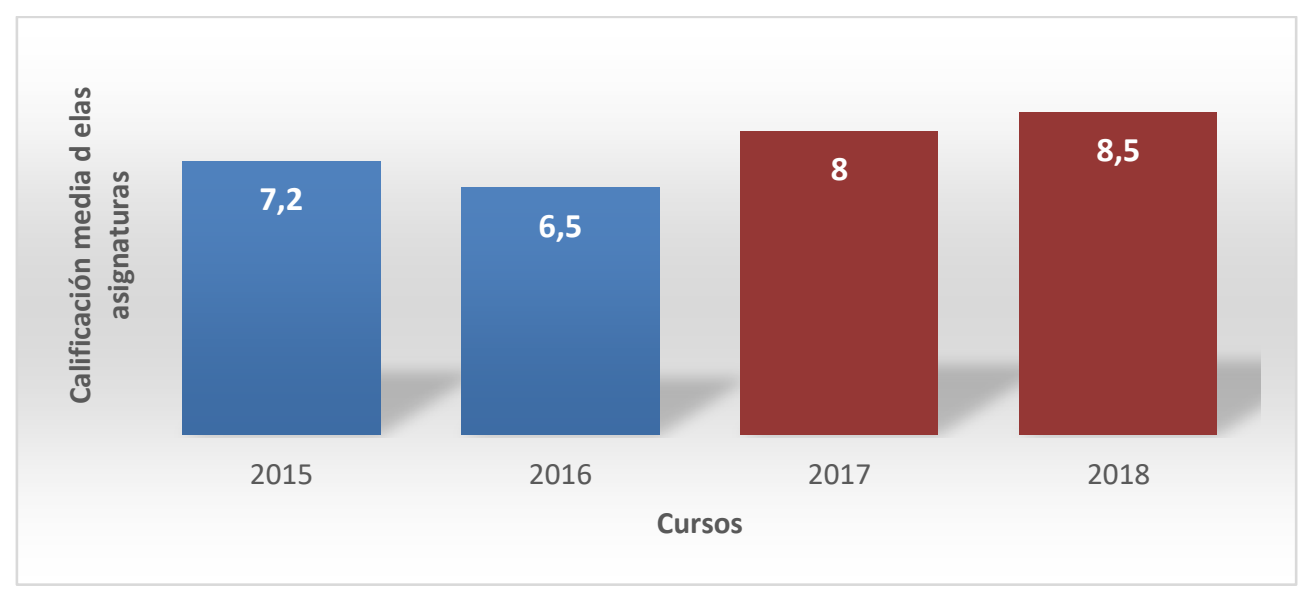

Figura 1. Calificación de las asignaturas a través de las encuestas institucionales (máxoma calificación 10) 
En la Figura 2 se muestra el porcentaje de alumnos aprobados por curso en cada uno de los años. 2017 y 2018, años donde se aplicó la metodología práctica (color rojo) y 2015 y 2016 (de color azul) donde la metodología aplicada fue convencional. El nivel de aprobados por curso aumentó alrededor de un $10 \%$.

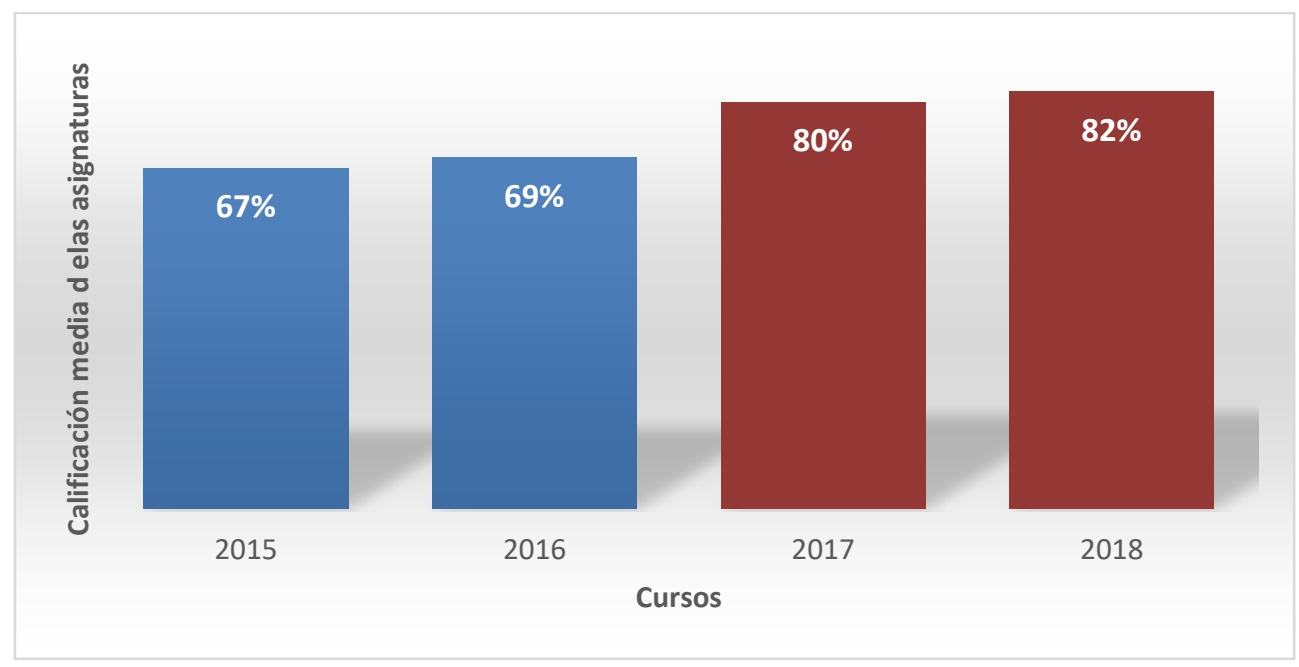

Figura 2. Porcentaje de alumnos aprobados por curso

Los resultados del análisis DAFO apuntaban a las siguientes direcciones

\section{Debilidades}

El funcionamiento de los foros no fue muy bien valorado. La mayoria de los alumnos no ha repasado los contenidos teóricos antes de la práctica. Eso obliga al profesor a hacer una disertación al estilo -elase magistral” apoyándose en una presentación.

La mayoría de los alumnos apuntaron que la realización de la evaluación mediante exámenes no les eximía de tener que recopilar textos para estudio de los contenidos teóricos.

Los alumnos con dispensa no desarrollan esta metodología, sino que sólo son evaluados con los exámenes parcial y final.

Entre las debilidades más relevantes se muestra la exigencia de ir a clase para seguir bien el método diseñado de aprendizaje. Si el alumno no puede asistir por alguna eventualidad inesperada no existia forma de recuperar la sesión práctica. 


\section{Amenazas}

Las infraestructuras de los centros educativos deben estar preparadas para un número de alumnos elevado en asignaturas que no son de especialidad.

La metodología no es tan aplicable en asignaturas básicas tales como matemáticas.

El número de aprobados por curso sí que es cierto que ha aumentado, pero el incremento es moderado.

Debe existir coordinación entre profesores de la misma asignatura y también coordinación con los profesores de otras asignaturas que también utilizan los mismos espacios para sus prácticas. Quizás sería inviable aplicar la misma metodología en todas las asignaturas de la carrera o del mismo curso.

\section{Fortalezas}

La organización de las asignaturas fue muy bien valorada, con un cronograma preciso, control de tiempos y espacios. También fue muy bien valorado el conjunto de materiales empleados tanto para la realización de las prácticas, como los recomendados para la preparación de los foro y studio de los contenidos teóricos.

La estructuración temática también fue bien valorada.

La metodología desarrollada provoca una fuerte motivación. El nivel de implicación en la asignatura es alto, incentivando el esfuerzo por dominar la técnica desde el punto de vista práctico.

Los contenidos teóricos siguen siendo abordados por los alumnos para la preparación del examen y también en las fichas a rellenar para las memorias de las prácticas.

\section{Oportunidades}

La percepción de los alumnos es que les prepara mejor para el ejercicio profesional. En consecuencia se ratifica el marco teorico por el cual la metodología ayuda a adquirir las cualidades personales o aptitudes y a las funciones metacognitivas por las cuales los egresados aumentan su seguridad en sus propias capacidades y confianza en sus conocimientos de base. 


\section{Conclusiones}

La metodología de enseñanza-aprendizaje basado en sesiones práctica resulta motivador para el alumnado. La percepción general es que vincula la formación con el ejercicio de la profesión, en este caso de ingeniería agronómica.

Esta metodología ofrece ciertas dificultades, principalmente vinculadas a la coordinación con otras asignaturas, necesidad de infraestructuras y preparación de los contenidos a desarrollar. También presenta dificultades en relación a que hay que establecer alternativas para los alumnos que no pueden asistir presencialmente a las clases y por ello poseen dispensa de asistencia.

La complementariedad de la docencia práctica con la realización de exámenes escritos se valora positivamente pues se evidencia que esto obliga a los alumnos a estudiar también contenidos teóricos con un ejercicio autónomo.

La evaluación de las prácticas mediante la entrega de memorias se valora positivamente porque establece un sistema de evaluación diversificado.

\section{Referencias}

Arandia Loroño, M.; Alonso-Olea, M.J. y Martínez-Domínguez, I. (2010). Ła metodología dialógica en las aulas universitarias. Revista de Educación, 352, 309-329.

Austin, J. E., y King, T. J. (2020). Applying Cultural-Historical Activity Theory to Understand the Development of Inclusive Curriculum Practices in Higher Education. International Journal of Inclusive Education, 24, 8: 882-900, https://doi.org/10.1080/13603116.2018.1492638.

Burke, P.J. y Manathunga C. (2020). The timescapes of teaching in Higher Education, Teaching in Higher Education, 25:6, 663-668, https://doi.org/10.1080/13562517.2020.1784618

Cohen, D. K. y Loewenberg Ball, D. (2007). Educational Innovation and the problem of scale. En B. Schneider y S. K. McDonald. Scale-up in education. Ideas in principle (pp. 19-36). USA: Rowman \& Littlefield Publishers, Inc.

Dollinger, M. (2020). The projectification of the university: consequences and alternatives. Teaching in Higher Education, 25(6), 669-682. https://doi.org/10.1080/13562517.2020.1722631

Jiménez, M., Caamaño, A., Oñorbe, A., y Pedrinaci, E. (2007). Los trabajos prácticos en ciencias. Barcelona, España: Graó.

Hundertmark, S, y Schanze, S. (2020). The Interplay Between Individual Reflection and Collaborative Learning - Seven Essential Features for Designing Fruitful Classroom Practices That Develop Students' Individual Conceptions." Chemistry Education Research and Practice : CERP., vol. 21, no. 3, University of Ioannina, , 765-88, https://doi.org/10.1039/c9rp00175a. 
Long, Y., Sun, D., Van Aalst, J., and Cheng, S.. (2020). Fostering Students' Creativity via Educational Robotics: An Investigation of Teachers' Pedagogical Practices Based on Teacher Interviews.” British Journal of Educational Technology. https://doi.org/10.1111/bjet.12985.

Loza Aguirre, M. (2004). Tertulias literarias. En: Cuadernos de pedagogía, 2004, p. 66-69.

Mikelli, D. (2020). Pedagogy of Difference 2.0: Interactive Documentary Practices and Participatory Research with Young People. Convergence-The International Journal of Research Into New Media Technologies. https://doi.org/10.1177/1354856520934724.

Pulido Mendoza, R., Aparicio Goñi, I., Mas Espejo, M., Tovar Reinoso, A., Rodríguez García, M. (2008). Entorno del aprendizaje práctico-clínico. Percepción de los estudiantes de Enfermería. V Jornadas de Innovacion Universitaria. Universidad Europea Madrid 4-5 septiembre 2008. http://hdl.handle.net/11268/3169

Rodríguez-Cepeda, R. (2016). Aprendizaje de conceptos químicos: una visión desde los trabajos prácticos y los estilos de aprendizaje. Revista De Investigación, Desarrollo e Innovación, 7(1), 6376. https://doi.org/10.19053/20278306.v7.n1.2016.4403

Sánchez Rodríguez, J. (2017). Preparación pedagógica, docentes clínicos de enfermería y su relación con el proceso-enseñanza aprendizaje práctico. Revista Cubana De EnfermeríA, 33(4). http://revenfermeria.sld.cu/index.php/enf/article/view/2131

Tsai, Y.-S., Rates, D., Moreno-Marcos, P. M., Muñoz-Merino, P. J., Jivet, I., Scheffel, M., Drachsler, H., Gašević, D., Delgado Kloos, C., Gašević, D. (2020). Learning analytics in European higher education-Trends and barriers. Computers \& Education, 155, 103933. https://doi.org/10.1016/j.compedu.2020.103933

Towers, J., Chapman, O., Drefs, M., y Friesen, S. (2020). Exploring the relationship between mathematics teachers' implicit associations and their enacted practices. Journal of Mathematics Teacher Education. Norwell, MA: https://doi.org/10.1007/s10857-019-09430-7 